\title{
Vorticity for the assessment of right ventricular diastolic dysfunction using 4D flow CMR
}

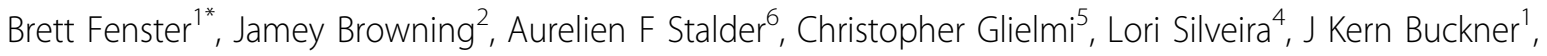 \\ Alex Kluiber $^{3}$, Joyce D Schroeder ${ }^{3}$, Jean Hertzberg ${ }^{3}$ \\ From 16th Annual SCMR Scientific Sessions \\ San Francisco, CA, USA. 31 January - 3 February 2013
}

\section{Background}

4D flow CMR analysis of right ventricular (RV) diastolic inflow has demonstrated vortical formations at tips of the tricuspid valve during the deceleration phase of early filling (E wave) (Figure 1). Vorticity can measure the rotation of these vortices and may represent a novel way to assess RV diastolic function. We aimed to determine if right heart vorticity identified right ventricular diastolic dysfunction (RVDD) in pulmonary hypertension (PH) subjects when compared to controls using $4 \mathrm{D}$ flow CMR.

\section{Methods}

Thirteen subjects ( 10 females/ 3 males) with $\mathrm{PH}$ and 10 age-matched controls ( 7 females $/ 3$ males) underwent same-day echocardiography and 4D flow CMR. Echocardiography assessed RV diastolic function and RV systolic pressure (RVSP). PH subjects had to demonstrate RVDD on the day of CMR as defined by either Stage I RVDD (tricuspid E/A < .8, E/E' $<6$, and deceleration time (DT) $>120 \mathrm{~ms}$ ) or Stage II RVDD (E/A > 2.1, E/E' of $>$ or $=6$, or DT $>120 \mathrm{~ms})$. 4D flow CMR was performed with interleaved 3-directional velocity encoding (spatial resolution $=3.5 \times 2.6 \times 3.0 \mathrm{~mm}^{3}, \alpha=15^{\circ}$, TE $/ \mathrm{TR}=2.85 / 48.56 \mathrm{~ms}$, venc $=150 \mathrm{~cm} / \mathrm{s}$, temporal resolution $=50 \mathrm{~ms}$ ) on a $1.5 \mathrm{~T}$ MRI system (Avanto, Siemens, Germany) using ECG gating and respiratory navigation. Images were acquired in a sagittal oblique 3D volume covering the entire right heart. Datasets were corrected for noise and aliasing using a custom Matlab program (Jelena Bock, Northwestern University) and imported into Paraview (Kitware, Clifton, NY) for isolation of the right heart field of view. Right heart E wave vorticity was calculated using Paraview. Univariate and multivariate regression analysis

were used to test the relationship between $\mathrm{E}$ wave vorticity and RVDD using JMP (SAS, Cary, NC). Heart rate and cardiac index were used to control for RV filling time and RV inflow, respectively.

\section{Results}

No significant difference in age or gender existed between control and RVDD groups (Table 1). RVSP was significantly elevated in RVDD vs. controls. All controls had normal RV diastolic function, while $6 \mathrm{PH}$ subjects had Stage I RVDD and 7 had Stage II RVDD. Mean E wave vorticity was significantly ( $p 0.006$ ) decreased in RVDD subjects $(0.31+/-0.11 / \mathrm{s})$ vs. controls $(0.47+/-0.17$ $1 / \mathrm{s})$. Decreased E wave vorticity correlated with RVDD presence ( $\mathrm{r} 0.66, \mathrm{p} 0.02$ ) and severity ( $\mathrm{r} 0.59, \mathrm{p} 0.001$ ) when controlled for age, gender, filling time, and RV inflow. E wave vorticity correlated with RVDD presence ( $\mathrm{r} 0.51, \mathrm{p} 0.01)$ and severity $(\mathrm{r} 0.37, \mathrm{p} 0.008)$ remained significant even in the absence of filling time and RV inflow.

\section{Conclusions}

Decreased right heart vorticity identifies the presence and severity of RVDD in a small cohort of PH subjects when compared to controls. Vorticity analysis for the assessment of RVDD may represent a novel clinical application of $4 \mathrm{D}$ flow CMR. Future investigations will require improvements in 4D flow CMR spatial and temporal resolution, larger patient cohorts, and simultaneous 4D flow CMR and echocardiographic acquisition to validate this finding.

\section{Funding}

Siemens Medical Solutions. 


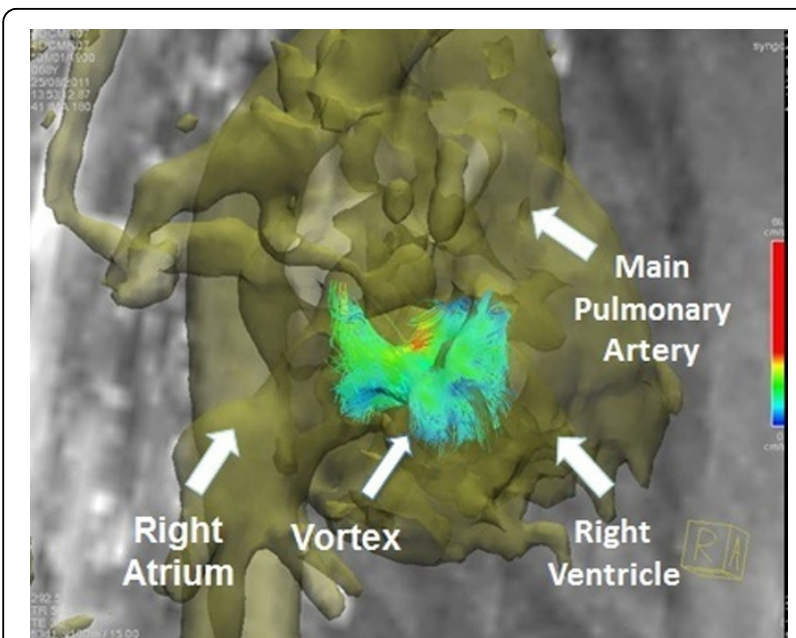

Figure $14 D$ CMR image of RV diastolic inflow with anatomic masking in a control subject. Image was taken in right anterior oblique view demonstrating vortex formation at the edge of an open tricuspid valve.

\section{Table 1 Patient cohort characteristics}

\begin{tabular}{cccc}
\hline & Control & RVDD & p Value \\
\hline Age (years) & $57+/-12$ & $63+/-6$ & NS \\
Gender (\% Female) & $70 \%$ & $77 \%$ & NS \\
RVSP (mmHg) & $58+/-17 \mathrm{mmHg}$ & $26+/-8 \mathrm{mmHg}$ & $\mathrm{p}<.001$ \\
\hline
\end{tabular}

NS $=$ not significant

\section{Author details}

'Division of Cardiology, National Jewish Health, Denver, CO, USA.

${ }^{2}$ Mechanical Engineering, University of Colorado, Boulder, CO, USA. ${ }^{3}$ Division of Radiology, National Jewish Health, Denver, CO, USA. ${ }^{4}$ Division of Biostatistics, National Jewish Health, Denver, CO, USA. ${ }^{5}$ Siemens Healthcare, New York, NY, USA. ${ }^{6}$ Magnetic Resonance, Imaging and Therapy, Healthcare Sector, Siemens AG, Erlangen, Germany.

Published: 30 January 2013

doi:10.1186/1532-429X-15-S1-P8

Cite this article as: Fenster et al:: Vorticity for the assessment of right ventricular diastolic dysfunction using 4D flow CMR. Journal of

Cardiovascular Magnetic Resonance 2013 15(Suppl 1):P8.

\section{Submit your next manuscript to BioMed Central and take full advantage of:}

- Convenient online submission

- Thorough peer review

- No space constraints or color figure charges

- Immediate publication on acceptance

- Inclusion in PubMed, CAS, Scopus and Google Scholar

- Research which is freely available for redistribution

Submit your manuscript at www.biomedcentral.com/submit 